\title{
Multi-Spacecraft Observations: Stream Interactions and Associated Structures
}

\author{
L.K. Jian • C.T. Russell · J.G. Luhmann • A.B. Galvin • \\ P.J. MacNeice
}

Received: 8 December 2008 / Accepted: 21 August 2009 / Published online: 3 October 2009

(c) The Author(s) 2009. This article is published with open access at Springerlink.com

\begin{abstract}
The stream interaction region (SIR), formed when a fast stream overtakes a preceding slow stream, is the predominant large-scale solar wind structure at this early phase of the STEREO mission. Using multi-spacecraft observations from STEREO A and B, ACE, Wind, and Ulysses in 2007, we analyze three stream interaction events in depth in May, August, and November of 2007, respectively, when the spacecraft had quite different spatial separations. We attempt to determine the causes of the differences in the SIR properties, whether they are spatial or temporal variations, and also to examine the steepening or widening of the SIR during its radial evolution. The presence and characteristics of associated shocks, the relation to the heliospheric current sheet, and other structures are also studied.
\end{abstract}

Keywords Solar wind $\cdot$ Stream interaction $\cdot$ Shock $\cdot$ Multi-spacecraft observation

STEREO Science Results at Solar Minimum

Guest Editors: Eric R. Christian, Michael L. Kaiser, Therese A. Kucera, O.C. St. Cyr

L.K. Jian $(\bowtie) \cdot$ C.T. Russell

Institute of Geophysics and Planetary Physics, University of California, Los Angeles, CA 90095, USA

e-mail: jlan@ igpp.ucla.edu

C.T. Russell

e-mail: ctrussel@igpp.ucla.edu

J.G. Luhmann

Space Sciences Laboratory, University of California, Berkeley, CA 94720, USA

e-mail: jgluhman@ssl.berkeley.edu

A.B. Galvin

Department of Physics, University of New Hampshire, Durham, NH 03824, USA

e-mail: toni.galvin@unh.edu

P.J. MacNeice

NASA, Goddard Space Flight Center, Code 674, Greenbelt, MD 20771, USA

e-mail: Peter.J.MacNeice@nasa.gov 


\section{Introduction}

Since slow and fast streams originate in different regions on the Sun at different times, they are threaded by different magnetic field lines and are prevented from interpenetrating (Gosling and Pizzo, 1999). Because the solar wind speed is not controled solely by heliographic latitude, as the Sun rotates, fast streams overtake preceding slow streams and outrun trailing slow streams. Hence, a pressure ridge forms on the leading portion of the fast stream and a rarefaction region forms at the trailing part.

Here, we are interested in the compression regions caused by stream interactions. These are usually characterized by significant enhancement of total perpendicular pressure $\left(P_{\mathrm{t}}\right)$, defined as the sum of the magnetic pressure and plasma thermal pressure perpendicular to the magnetic field (Russell, Shinde, and Jian, 2005) with a relatively gradual decline at both sides of the maximum. Within the stream interaction region (SIR), the plasma is compressed, deflected, and heated. The interaction drives pressure waves at the leading and trailing edges of the SIR, which can steepen into shocks as the compression intensifies (e.g., Hundhausen and Gosling, 1976; Smith and Wolfe, 1976). Based on hundreds of events throughout almost complete solar cycles, Jian et al. $(2006,2008)$ found that the shock association rate increases from about $3 \%$ to $26 \%$ from 0.72 to $1 \mathrm{AU}$, indicating the region near $1 \mathrm{AU}$ is an incubator for SIR-driven shocks. We will analyze the occurrence and characteristics of SIR-driven shocks at various spacecraft in this article.

The difference between a SIR and a corotating interaction region (CIR) is that the SIRs include not only CIRs that persist on successive Carrington rotations (CRs), but also transient or localized SIRs. Because the coronal source regions of the fast and slow streams can sometimes change dramatically within one solar rotation when the Sun is very active, the resulting interaction regions can become transient and do not form a corotating pattern lasting more than one solar rotation (Balogh et al., 1999). The events focused herein all occurred in 2007, near solar minimum, and are all CIRs.

After the launch in October 2006, the twin Solar-Terrestrial Relations Observatory (STEREO) spacecraft entered approximately 1 AU heliocentric orbits in the ecliptic plane, drifting away from the Earth at a rate of about $20^{\circ}$ per year in opposite directions (Kaiser et al., 2008). Considering the increasing longitudinal separation of the twin spacecraft, we choose to study three SIRs, occurring in May, August, and November 2007, respectively, in order to understand their spatial and/or temporal variations. Due to the radial propagation of SIRs and the solar rotation, the difference between the arrival time of an SIR at spacecraft 1 and at spacecraft 2 would be

$$
\Delta t=t_{2}-t_{1}=\left(\varphi_{2}-\varphi_{1}\right) / \omega_{\text {Sun }}+\left(r_{2}-r_{1}\right) / v_{\mathrm{sw}},
$$

where $\varphi$ denotes the longitude, $\omega_{\text {Sun }}$ the solar rotation angular speed, $r$ the heliocentric distance, and $v_{\mathrm{sw}}$ the solar wind speed. This equation will be used to analyze the following events.

For STEREO, we use the 1-min time resolution data from the following two instrument suites: In-situ Measurements of Particles and CME Transients (IMPACT, Luhmann et al., 2008), as well as the Plasma and Suprathermal Ion Composition (PLASTIC, Galvin et al., 2008). To analyze some fine-scale structures, such as shocks, the $8 \mathrm{~Hz}$ magnetic field data from the Magnetic Field Experiment (Acuña et al., 2008) are also used.

Besides the STEREO A (STA) and B (STB) data, the solar wind data from Wind, Advanced Composition Explorer (ACE), and Ulysses are also approached. Both Wind and ACE data are needed in order to provide good data coverage for August and November near-Earth cases. 
From the Wind spacecraft, we mainly use the 93-sec resolution data from the Solar Wind Experiment (SWE, Ogilvie et al., 1995) and the Magnetic Fields Investigation (MFI, Lepping et al., 1995). To identify shocks, higher time resolution data of SWE $(6-12 \mathrm{sec})$, MFI (3-sec), and 3-D Plasma Analyzer (24-sec, Lin et al., 1995) are used. From the ACE spacecraft, we use the validated Level 2 data from the Solar Wind Electron, Proton, and Alpha Monitor (SWEPAM, McComas et al., 1998) and the Magnetic Field Experiment (Smith et al., 1998), both at 64-sec time resolution. The Level 2 magnetic field data at 16-sec resolution are used to identify shocks.

After the solar south pole passage in March 2007, Ulysses started to move northward. It passed the solar equatorial plane on 11 August and the ecliptic plane on 19 August in 2007. This perihelion pass provides a good opportunity to study the radial evolution of an SIR from 1 to $1.4 \mathrm{AU}$, which will be addressed later in this article by investigating the August event. We use the 4-min resolution plasma data from the Solar Wind Observations Over the Poles of the Sun (SWOOPS) instrument (Bame et al., 1992) and magnetic field data from the magnetometer (Balogh et al., 1992) onboard the Ulysses spacecraft. To identify shocks, 1 -sec resolution magnetic field data are also used.

\section{Multi-Spacecraft Observations}

\subsection{STA and STB: $7-8$ May 2007}

Based on the photospheric magnetograph from the Mount Wilson Observatory (MWO, Ulrich et al., 2002), the Wang - Sheeley - Arge (WSA) coronal model (Arge et al., 2004, and references therein) can derive the coronal field and solar wind speed at 21.5 solar radii $\left(R_{\mathrm{S}}\right)$. Figure 1 illustrates such a case for CR 2055 longitude $60^{\circ}-$ CR 2056 longitude $60^{\circ}$. Considering the solar wind travel time, we confine the interval of 2-6 May by the blue dashed lines, as the photospheric and coronal conditions for the solar wind observed near the Earth during 7-8 May. Because of the speed difference in the slow and fast streams, the confined duration in Figure 1 is longer than the duration in Figure 2. As illustrated in the bottom panel, the fast solar wind during these two days should be primarily associated with a lowlatitude coronal hole in the southern hemisphere, also visible from the STEREO Extreme Ultraviolet Imager (EUVI) image (Howard et al., 2008) at that time.

During 7-8 May 2007, the twin STEREO spacecraft were separated by about $0.09 \mathrm{AU}$ in the radial direction. Their latitudinal and longitudinal offsets are $1^{\circ}$ and $7^{\circ}$, respectively. From Equation (1), STA should encounter the SIR about $3 \mathrm{~h}$ later than STB, but both the forward shock and heliospheric current sheet (HCS) arrived $3 \mathrm{~h}$ earlier at STA than at STB. This implies that the plasmas encountered by the two spacecraft may have started from the Sun at different times.

From the polarity changes of $B_{\mathrm{r}}$ (blue dashed line) and $B_{\mathrm{t}}$ (green dotted line) in the sixth panel of Figures 2(a) and 2(c), we can discern the HCS, as marked by the black solid vertical line. This is also confirmed by the change of the suprathermal-electron pitch angle distribution (not shown) from IMPACT Solar Wind Electron Analyzer (SWEA, Sauvaud et al., 2008). Since the coronal field neutral line was about $10^{\circ}$ north in Figure 1 (1), the crossing of HCS at STA and STB, both in the southern hemisphere, indicates a moderate inclination of the HCS. At both spacecraft, the HCS crossing occurred about $2 \mathrm{~h}$ after the forward shock, which is not the most common case for 1-AU SIRs because the HCS usually leads the SIR at such a heliocentric distance and becomes embedded within the SIR later as the SIR expands along with its outward propagation. The HCS location relative to the SIR 


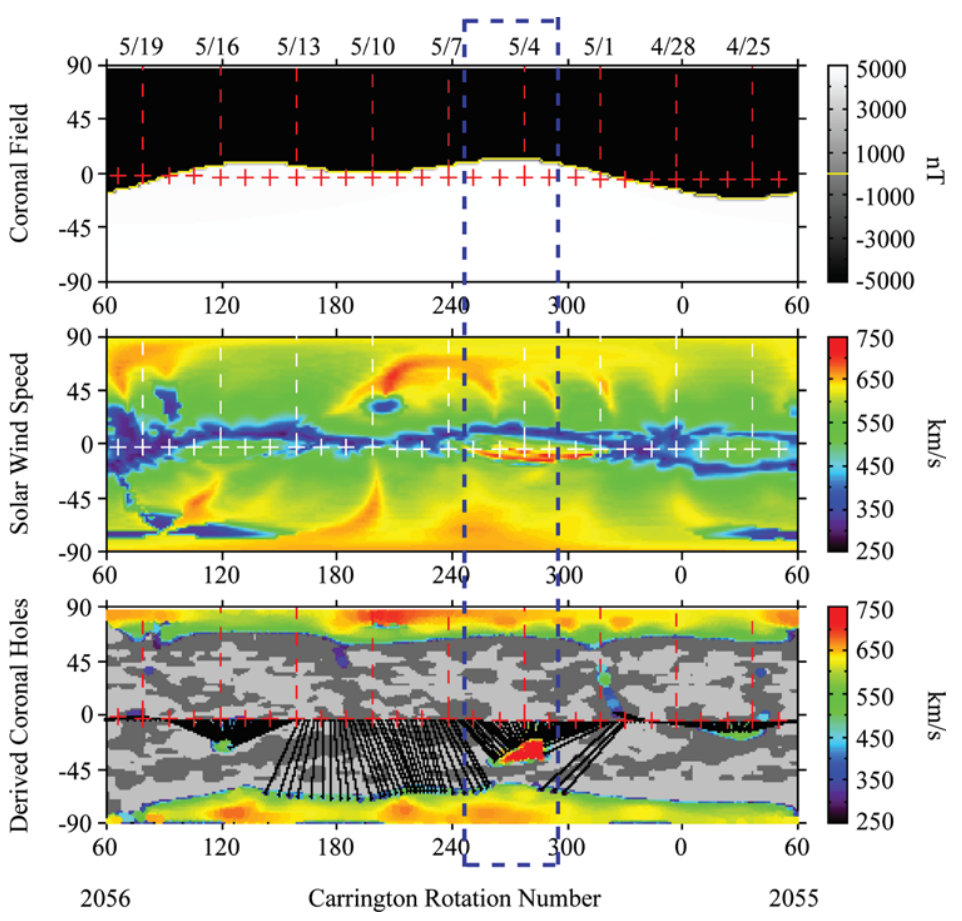

Figure 1 The coronal sources derived from the WSA coronal model based on the MWO photospheric magnetograph, covering the region CR 2055 longitude $60^{\circ}-\mathrm{CR} 2056$ longitude $60^{\circ}$, with time sequence from right to left. The blue dashed lines bound the approximate interval of $2-6$ May. The panels from top to bottom: the calculated global coronal field polarity at $21.5 R_{\mathrm{S}}$, where the yellow solid curved line indicates the neutral sheet between the inward and outward field lines; the derived solar wind speed at $21.5 R_{\mathrm{S}}$; the derived coronal hole areas, with the solid black lines connecting the outer coronal boundary at $21.5 R_{\mathrm{S}}$ and its source regions at the photosphere. In other words, the colored dots represent photospheric footpoints of the open field lines. The areas shaded light (or dark) gray are closed field lines with $B_{\mathrm{r}}>0$ (or $B_{\mathrm{r}}<0$ ) in the photosphere. The color scale indicates the solar wind speed at $21.5 R_{\mathrm{S}}$ (related to the expansion factor, see Arge et al., 2004, and references therein) associated with the flux tubes. In all three panels, the + symbol marks the daily position of the sub-Earth point on the Sun. Because the solar rotation axis tilts with respect to the ecliptic plane, the sub-Earth point is not exactly at the solar rotational equator, and it can vary between $\pm 7.25^{\circ}$ in latitude. Courtesy of Community Coordinated Modeling Center (CCMC).

in this event is probably due to the HCS tilt and the forward shock expansion. Several such cases were found in our STEREO survey.

The SIR drove a forward shock at both spacecraft and also a reverse shock at STA, all of which are indicated by red dotted vertical lines. At STB, the variations of $N_{\mathrm{p}}$ and $B$ at 14:11 UT on 8 May mimic the signatures of a reverse shock; however, the changes of $T_{\mathrm{p}}$ and $S$ indicate it was not a shock otherwise the downstream plasma would be heated.

Using 8-Hz magnetic field data, we can well determine the parameters of these shocks. The shock normal angles of the forward shock were $62^{\circ}$ and $80^{\circ}$ at STB and STA, respectively. In RTN coordinates, the directions of the forward shocks at STB and STA were $[0.86 \mathbf{R}, 0.49 \mathbf{T}, 0.13 \mathbf{N}]$ and $[0.81 \mathbf{R}, 0.51 \mathbf{T},-0.27 \mathbf{N}]$, respectively, where $\mathbf{R}$ is the unit vector from the Sun to spacecraft, $\mathbf{T}$ being $(\boldsymbol{\Omega} \times \mathbf{R}) /|(\boldsymbol{\Omega} \times \mathbf{R})|$ with $\boldsymbol{\Omega}$ as the Sun's spin axis, and $\mathbf{N}$ completing the right-handed triad. So they were both antisunward and westward, consistent with Gosling et al. (1993b). However, their north-south directions were opposite, which is odd because they should be on the same side of the HCS. It may be at- 

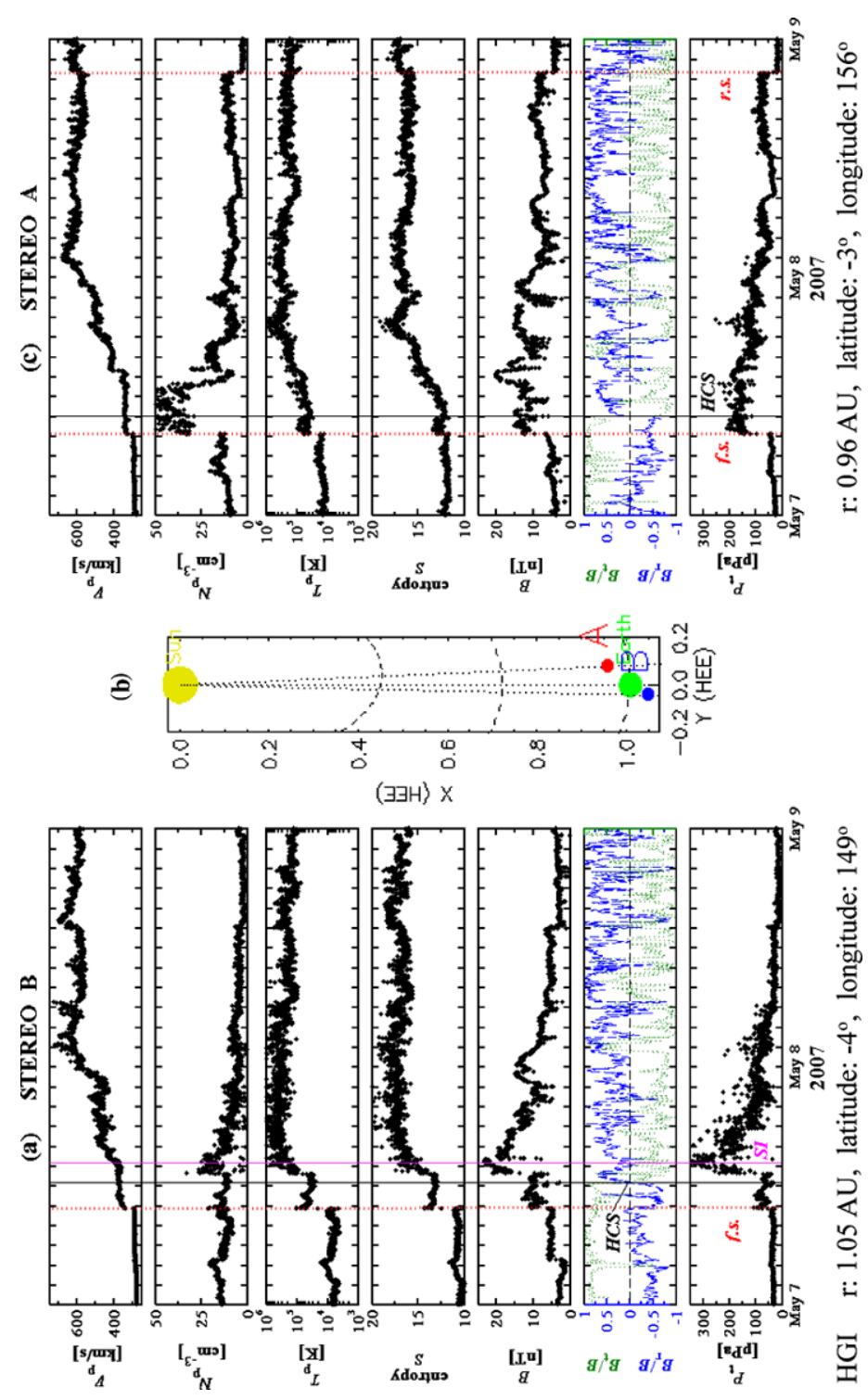

苛

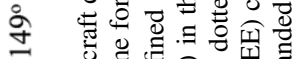

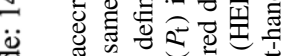
O

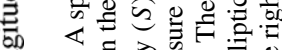

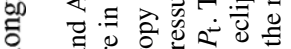
สิ +ं

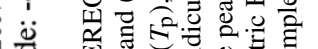

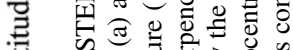

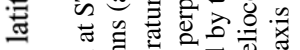

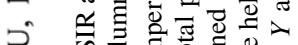

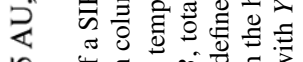
๖ i.

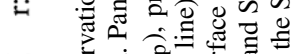
ত

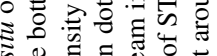

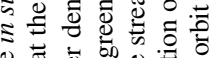

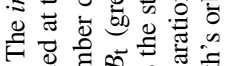

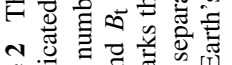

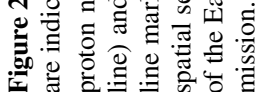


tributed to some small-scale solar wind variations nearby. Nevertheless, this implies that the direction of a SIR-driven shock is sensitive to location. The reverse shock at STA was quasi-parallel, with a shock normal angle of $25^{\circ}$. Its direction in RTN coordinates was $[-0.82 \mathbf{R},-0.56 \mathbf{T},-0.10 \mathrm{~N}]$, suggesting this shock normal was mostly confined in the ecliptic plane, sunward and eastward.

The parameter variations across the forward shocks differed: the $T_{\mathrm{p}}$ and $S$ increased more at STB than at STA, while the $N_{\mathrm{p}}$ and $P_{\mathrm{t}}$ increased more at STA than at STB. The remarkable differences of the forward shock at the two nearby spacecraft and the nonexistence of the reverse shock at STB suggest that SIR-driven shocks were still in development at $1 \mathrm{AU}$ and they were limited in extent.

The SIR at STB lasted about $7 \mathrm{~h}$ shorter than at STA. The $V_{\mathrm{p}}$ varied similarly at the two locations, while the $N_{\mathrm{p}}, T_{\mathrm{p}}, S, B$, and $P_{\mathrm{t}}$ varied fairly differently at the two spacecraft, especially the $N_{\mathrm{p}}$ and $P_{\mathrm{t}}$. At STA, during 08:00-13:00 UT on 7 May, the $N_{\mathrm{p}}$ remains near 50 $\mathrm{cm}^{-3}$, at least two times denser than other regions. The absence of such a density enhancement at STB and the almost constant inclination of the coronal field neutral line in the top panel of Figure 1, imply the dense region is unlikely to be due to the heliospheric plasma sheet (Winterhalter et al., 1994) surrounding the HCS, but more likely due to nonuniform compression as the streams interact. At STB, the $P_{\mathrm{t}}$ profile is in a very asymmetric shape. Its peak marks the stream interface (SI), as it indicates where the forces at the two sides are balanced (Jian et al., 2006). At STB, the $P_{\mathrm{t}}$ increased by about $300 \mathrm{pPa}$ within $1 \mathrm{~h}$ at about 13:10 UT on 7 May, and returned to the background level one day later. Thus, the force pushing toward the leading slow stream is much stronger than the force pushing toward the trailing portion of the fast stream, although the integrated force, the pressure, is the same.

At STB, the $N_{\mathrm{p}}, T_{\mathrm{p}}, S, B$, and $P_{\mathrm{t}}$ all increased abruptly at 7 May, 13:10 UT, although it was not a shock as the $V_{\mathrm{p}}$ did not increase. In contrast, there was no such concurrent variation of multiple parameters within the SIR at STA. The $T_{\mathrm{p}}$ and $S$ increased gradually between the two streams at STA, implying some dissipation might exist in that region. Moreover, the $P_{\mathrm{t}}$ profile of the SIR at STA was like a plateau with a gradual decline toward the trailing portion, rather than the ideal shape of an SIR - a pile-up with gradual declines at the two sides. Overall, although their locations were close, the differences observed at STA and STB were surprisingly large. These may be attributed to the temporal variations of the plasma source region.

\subsection{STB, Wind, STA, and Ulysses: 5 - 10 August 2007}

As mentioned in the Introduction, Ulysses passed the ecliptic plane on 19 August 2007. During its perihelion pass, only three SIRs were observed by multiple spacecraft when Ulysses was within a $10^{\circ}$ latitudinal offset from the ecliptic plane. We choose the 9 August event because it is the most representative one among the three. The projection of the Ulysses trajectory and the positions of STEREO A and B as well as nearby planets in the ecliptic plane are all sketched in Figure 3.

Figure 4 illustrates the calculated solar wind speed and coronal field using the WSA coronal model for the region CR 2059 longitude $320^{\circ}$ - CR 2060 longitude $320^{\circ}$, based on the MWO photospheric field. The blue dashed lines confine the coronal conditions for the solar wind observed near the Earth during 5-8 August. As shown in the first panel, the inclination of the field neutral line was constant during this interval. This is also consistent with the distribution of the slow wind region (dark blue) in the second panel because the slow wind usually originates from the streamer belt surrounding the field neutral line (e.g., Feldman et al., 1981; Gosling et al., 1981). In addition, from the derived foot-points of the 
Figure 3 The projections of STA (red dot), STB (blue dot), Ulysses trajectory (magenta dot and magenta dashed line), and the orbits of nearby planets in the HEE plane on 9 August 2007.

The distances are in the unit of AU. Courtesy of the STEREO mission.
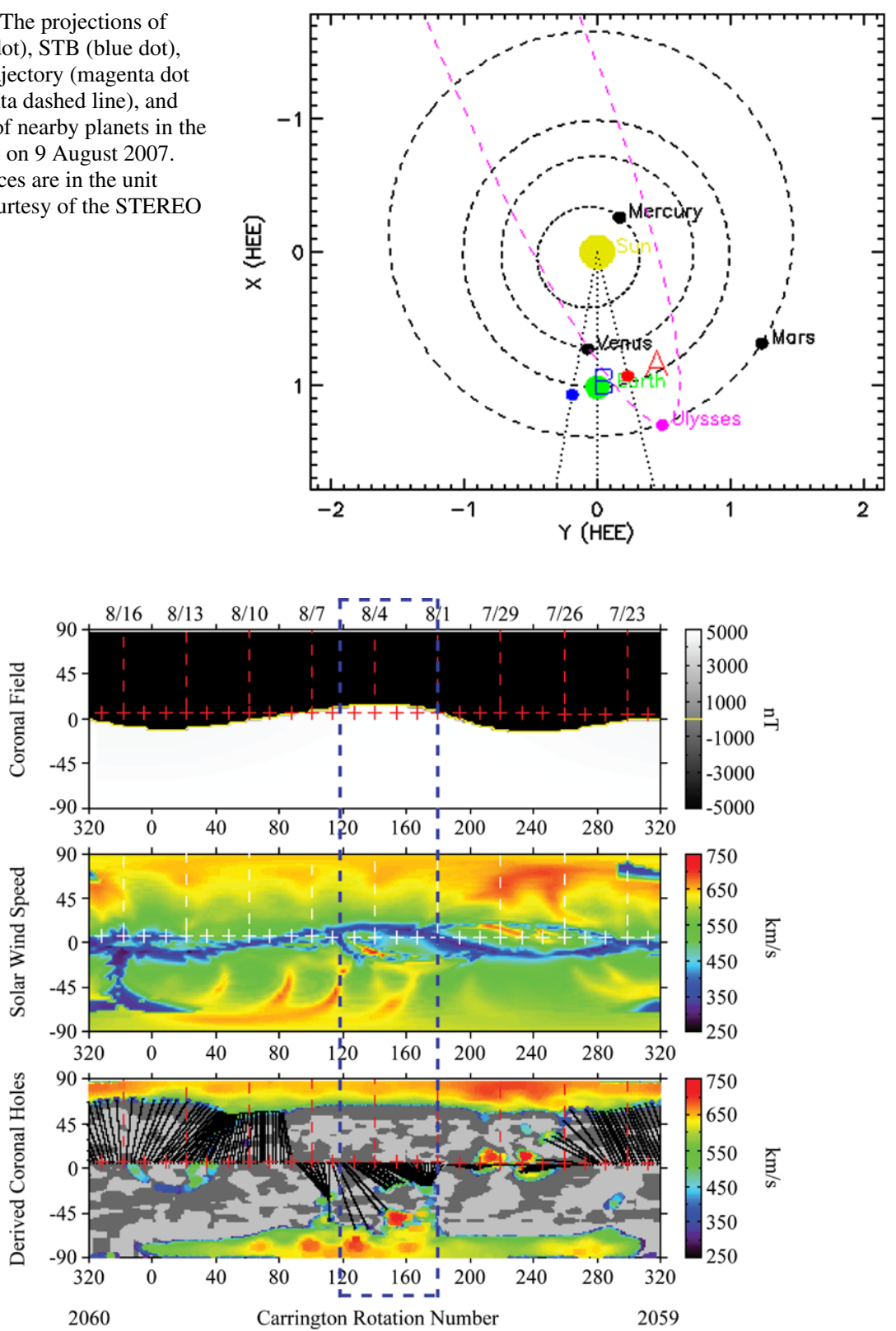

Figure 4 The coronal sources derived from the WSA coronal model based on the MWO photospheric magnetograms, covering the region of CR 2059 longitude $320^{\circ}-\mathrm{CR} 2060$ longitude $320^{\circ}$. The blue dashed lines bound the interval $1-5$ August. The figure captions of Figure 1 apply. Courtesy of CCMC.

open field lines at the bottom panel, we can see that the open field lines during the confined interval are mainly connected with a middle-latitude small coronal hole.

The three columns of Figure 5 display the in situ observations for a same SIR at the STB, Wind, and STA spacecraft, respectively, in a similar format to Figure 2. The black solid 

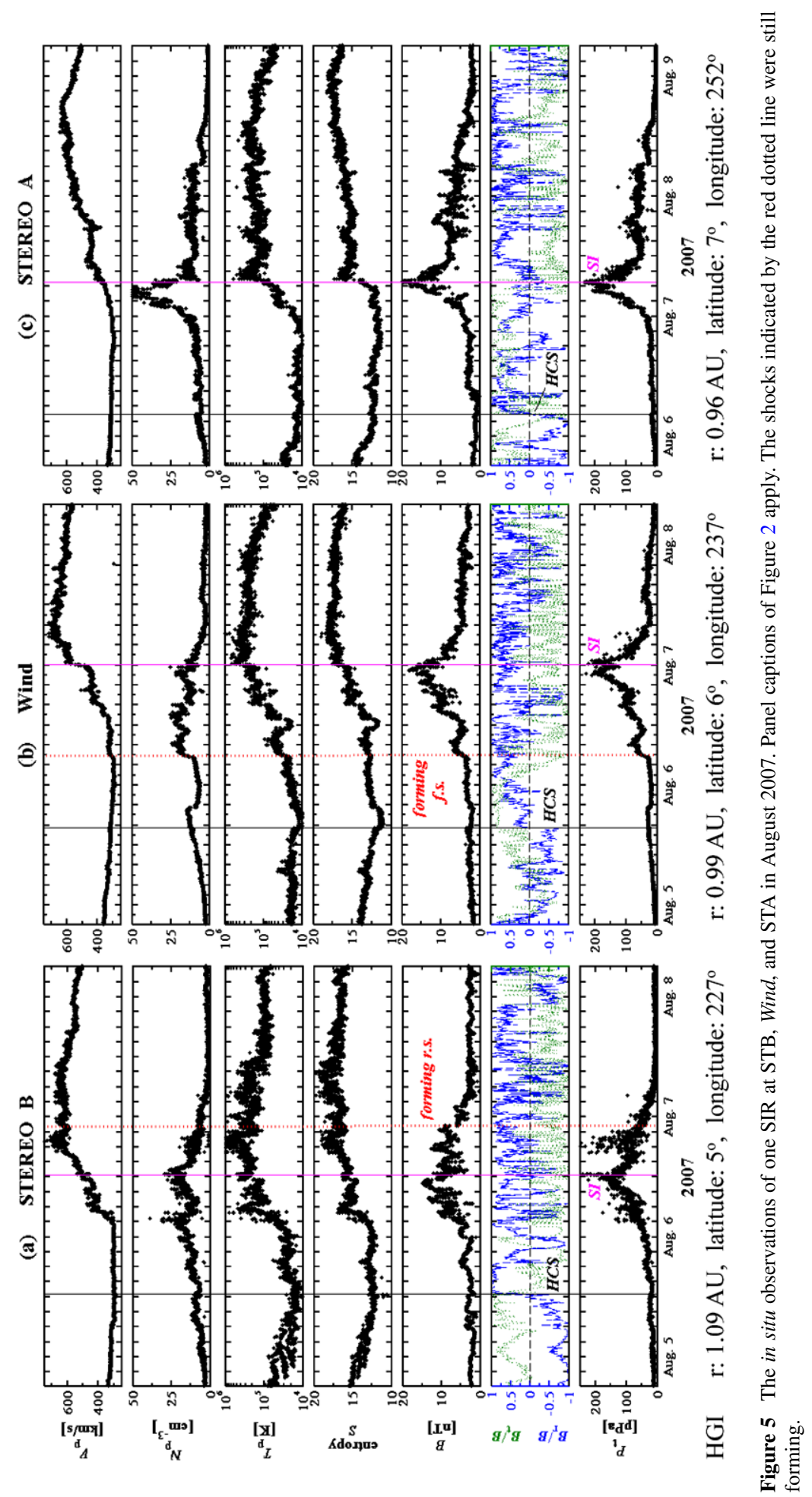
line indicates the HCS crossing, confirmed by the pitch angle distribution of suprathermal electrons (not shown here), and the magenta solid line marks the stream interface defined by the $P_{\mathrm{t}}$ peak. In contrast to the May event, the HCS was ahead of the SIR at all three spacecraft, at least $20 \mathrm{~h}$ before the SI. Hence, the variations within this SIR should not be related to the heliospheric plasma sheet at all.

Although the STA spacecraft was about 0.13 AU closer to the Sun than the STB, it was $25^{\circ}$ west of STB, so it observed the SIR about one day later than STB, as expected from Equation (1). In contrast with STB and Wind, the SIR observed at STA had a more gradual $V_{\mathrm{p}}$ increase, stronger $N_{\mathrm{p}}$ and $B$ compression, and a sharper $T_{\mathrm{p}}$ and $S$ increase. It is not easy to determine whether spatial or temporal effects played a more important role in causing these variations. The $P_{\mathrm{t}}$ profile displayed differently at the three spacecraft. It was symmetric at STB and became asymmetric at Wind and STA, one with a long leading portion, the other with a long trailing part. A reverse shock was forming at STB, with a sharp change of $B$ and no abrupt changes of $N_{\mathrm{p}}$ and $T_{\mathrm{p}}$; while a forward shock was forming at Wind, with sharp changes of $V_{\mathrm{p}}, N_{\mathrm{p}}$, and $T_{\mathrm{p}}$, but only a gradual $B$ increase. Neither of the forming shocks are seen at STA. All of the above facts imply that the forces acting at the two sides of the stream interface, and also the shocks driven by such forces, were fairly variable and somewhat transient.

At Wind and STA, a traditionally defined stream interface, characterized by an abrupt drop in $N_{\mathrm{p}}$ with simultaneous rises in $T_{\mathrm{p}}$ and $S$ (e.g., Belcher and Davis, 1971; Gosling et al., 1978), can be discerned. It coincides with the location at which $P_{\mathrm{t}}$ maximized as expected. Since, at present, no full velocity vector is widely available from STEREO, we do not consider the flow shear criterion here. The absence of the traditional signatures of a $\mathrm{SI}$ at STB, at a greater heliocentric distance, along with the increasing abrupt variations of $N_{\mathrm{p}}$ and $B$ from STB to STA, demonstrates the variability of the transition between slow and fast streams. This might be attributed to the temporal variation of the plasma source region and/or spatial offsets. In addition, the SIR observed at Wind was not simply a transition from STB to STA, so it is also possible that the sharp boundary between slow and fast streams is not a consistent or large-scale structure.

As shown in Figure 3, of the three spacecraft STB, Wind, and STA, the STA was closest to Ulysses during this event. So, in Figure 6, we compare the observations at STA and Ulysses with an $8^{\circ}$ latitudinal offset between them. At Ulysses, a pair of forward-reverse shocks formed at the two edges of the SIR, although the two shocks were still weak there, with small changes of plasma and field parameters across them. This demonstrates the region close to $1 \mathrm{AU}$ is indeed an incubator for SIR-driven shocks (Russell et al., 2009). The HCS was closer to the SI at Ulysses than at STA, likely due to latitudinal differences.

The SIR lasted a shorter time at Ulysses than at STA, though with a similar solar wind speed. The shock pair at Ulysses indicates the SIR is expanding there. Hence, the smaller size at Ulysses than at STA is likely due to latitudinal differences. In addition, there was no traditionally defined SI at Ulysses. Although $N_{\mathrm{p}}, B$, and $P_{\mathrm{t}}$ data of Ulysses are not rescaled by $R^{2}$ to $1 \mathrm{AU}$, we can still see the trend of the variation from their temporal profiles. Generally, the variations of $N_{\mathrm{p}}, T_{\mathrm{p}}, S$, and $B$ were all more gradual at Ulysses than at STA, again indicating the compression effect of the stream interaction was not dominant. There were drops of temperature and entropy lasting about $5 \mathrm{~h}$ before the SI, the cause of which is unclear. Nevertheless, the various changes in the shown parameters should be caused by a combination of the compression between streams, the expansion led by shocks, the dissipation within SIR, and the latitudinal offset.

The suprathermal electron fluxes were enhanced within the SIR at both spacecraft, and there were counterstreaming suprathermal electrons (CSEs) at Ulysses upstream of the re- 
(a) STEREO A at $1 \mathrm{AU}$
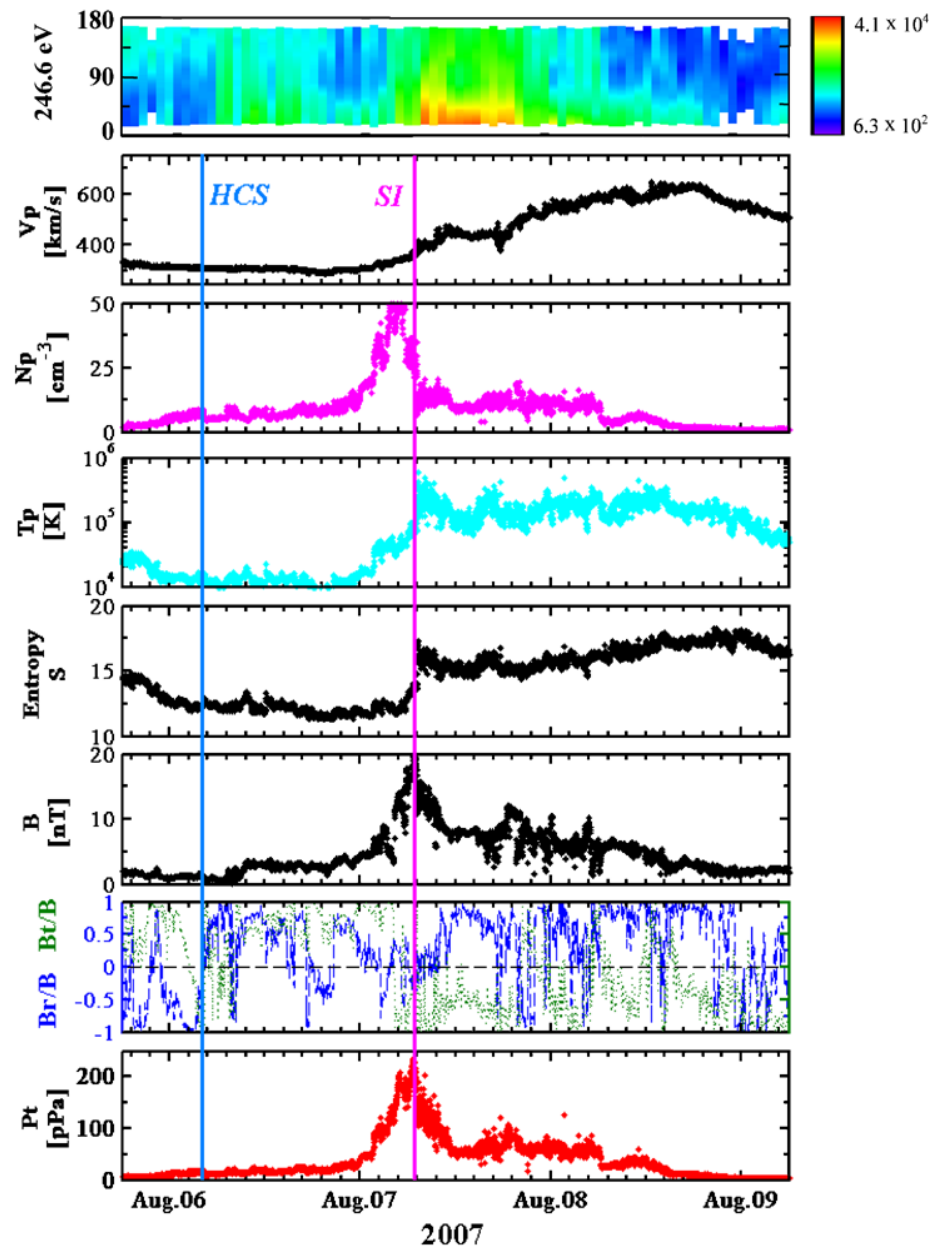

HGI latitude: $7^{\circ}$, longitude: $252^{\circ}$

Figure 6 The observations of the SIR in Figure 5 at STA and Ulysses. The first panel shows the pitch angle spectrogram of suprathermal electrons. Other panels display the same parameters as in Figure 2. The blue solid vertical line indicates the HCS. The magenta solid vertical line marks the SI. The red dotted line indicates the forward and reverse shocks.

verse shock, although not upstream of the forward shock. The CSEs lasted over $30 \mathrm{~h}$ upstream of the reverse shock. They were probably produced when electrons, energized at the reverse shock, subsequently leaked out of the SIR into the upstream solar wind (e.g., Gosling et al., 1993a; Steinberg et al., 2005, and references therein).

\subsection{STB, ACE, STA: 12 - 15 November 2007}

In November 2007, the longitudinal separation between the STEREO twin spacecraft increased to about $42^{\circ}$. We investigate in depth one representative SIR observed by multiple spacecraft near $1 \mathrm{AU}$ as follows. 
(b) Ulysses at 1.4 AU

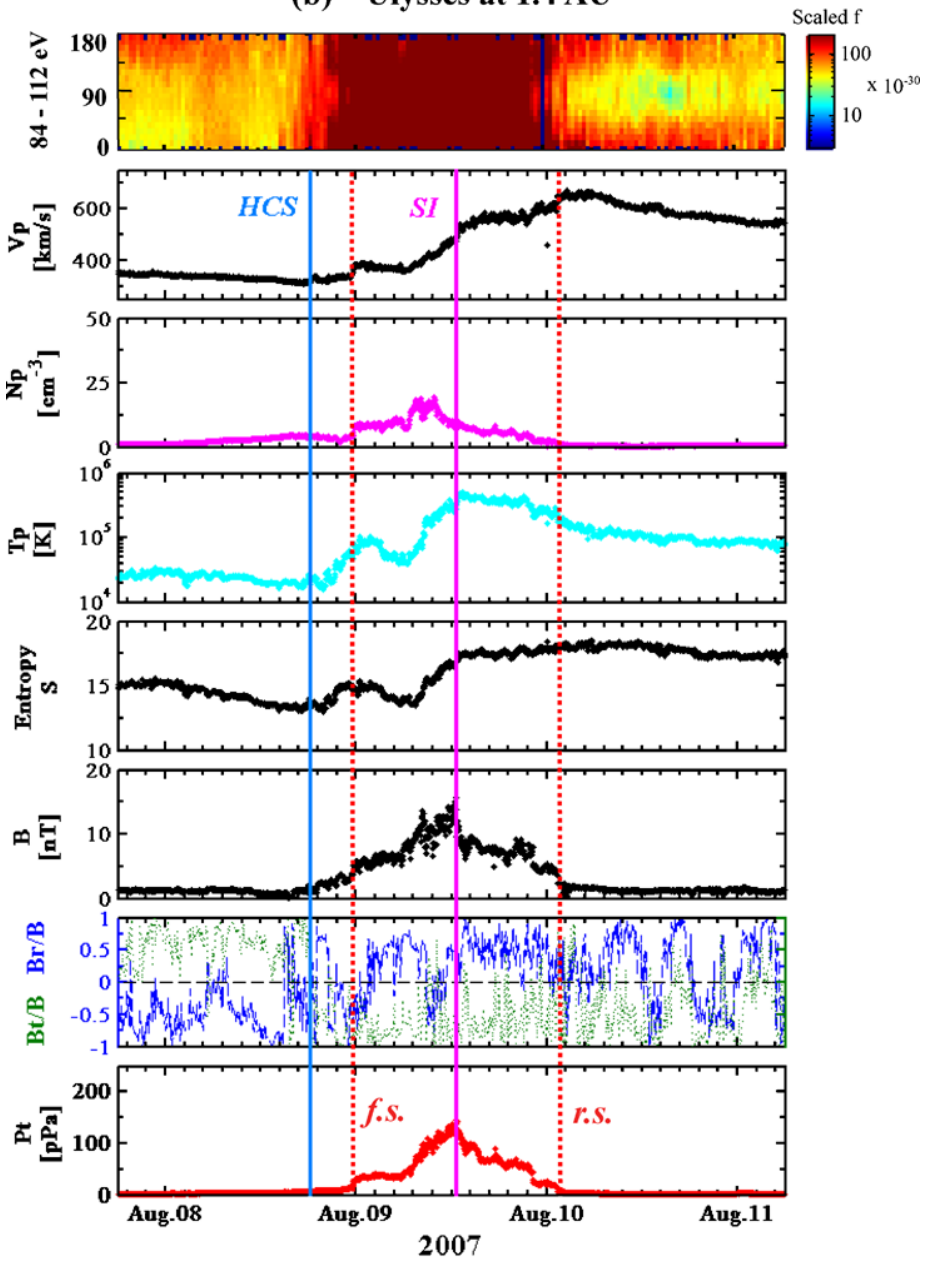

HGI latitude: $-2.4^{\circ}$ to $-0.9^{\circ}$, longitude: $260.7^{\circ}$

Figure 6 (Continued.)

Figure 7 shows the coronal field and solar wind derived by the WSA coronal model based on data from the MWO photospheric magnetograph, for the region CR 2062 longitude $40^{\circ}$ CR 2063 longitude $40^{\circ}$. The blue dashed lines outline the coronal conditions for the solar wind observed near the Earth during $12-15$ November. As illustrated in the bottom panel, the fast wind during this interval predominantly originated from the polar coronal hole.

The in situ observations of the SIR at the STB, ACE, and STA spacecraft are compared in Figure 8. No HCS was crossed near the SIR. Within the SIR, the suprathermal electron fluxes increased, similar to the August event. Because the energy level and distribution function available from ACE and STEREO are different, we only compare the fluxes at STB and STA in the following. The flux was higher and covered a larger pitch angle range at STA than at STB, possibly due to a larger amount of heating associated with the stronger compression within the SIR at STA than at STB. As the SIR evolved, the trailing edge 

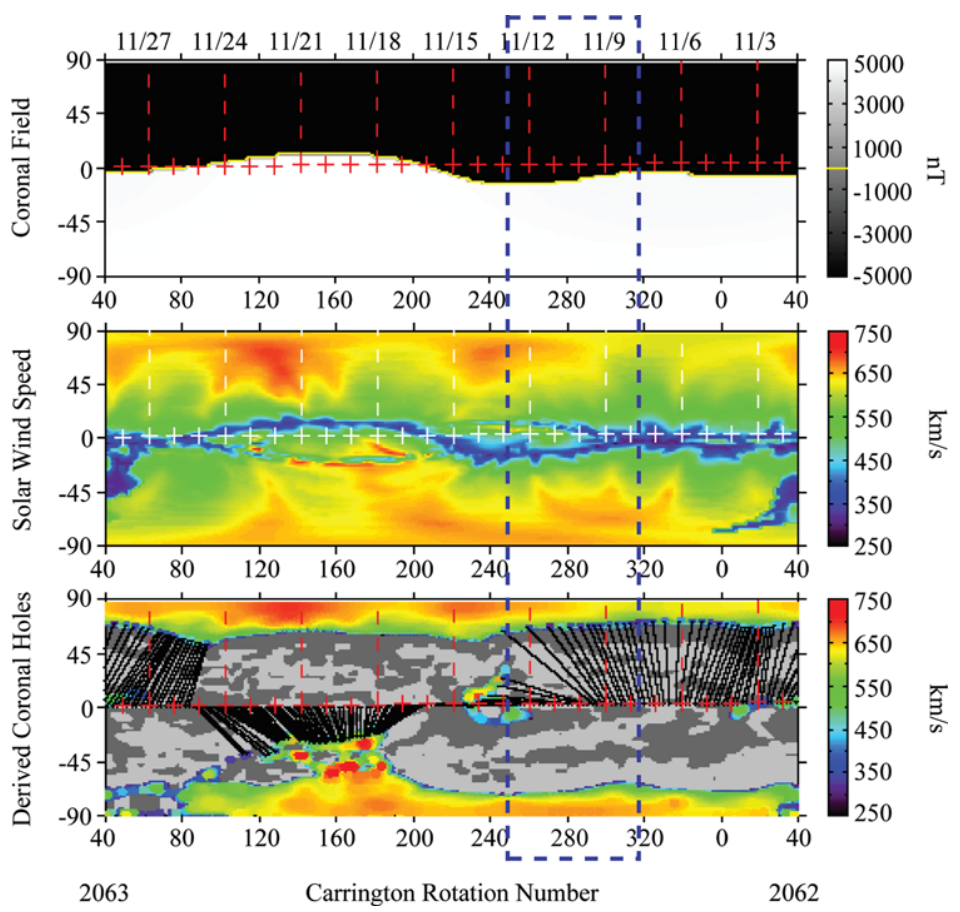

Figure 7 The coronal sources derived from the WSA coronal model based data from the MWO photospheric magnetograph, covering the region CR 2062 longitude $40^{\circ}-\mathrm{CR} 2063$ longitude $40^{\circ}$. The blue dashed lines bound the interval 7-12 November. The figure captions of Figure 1 apply. Courtesy of CCMC.

steepened into a reverse shock at STA. This shock is oblique, with a shock normal angle of $65^{\circ}$, and a magnetosonic Mach number of about 1.3. The shock normal direction is about $[-0.80 \mathbf{R},-0.15 \mathrm{~T},-0.59 \mathrm{~N}]$ in RTN coordinates, i.e., sunward, eastward, and southward. Upstream of this reverse shock, there were some suprathermal electrons at $0^{\circ}$ pitch angle, i.e., along the magnetic field, but the flux in this direction was not intense and did not last long either. Comparing it with the clear CSEs at Ulysses in the August event, we speculate that counterstreaming of electrons has not yet begun inside of $1 \mathrm{AU}$.

At STB, the enhanced regions of $N_{\mathrm{p}}, B$, and $P_{\mathrm{t}}$ lasted more than one day. We cannot discern a stream interface using the $P_{\mathrm{t}}$ peak, because the $P_{\mathrm{t}}$ profile was like a plateau. The absence of a pressure ridge feature implies that the interaction between slow and fast streams might have not yet grown strong. However, there was a traditionally defined stream interface at about 09:00 UT on 13 November, although the decrease of $N_{\mathrm{p}}$, as well as the increases of $V_{\mathrm{p}}, T_{\mathrm{p}}$, and $S$ were not very abrupt. The gradual entropy increase from slow to fast stream, i.e., from the beginning of 12 November to the middle of 13 November, indicates the existence of dissipation between them.

At ACE, $20^{\circ}$ west of STB, the compression of $N_{\mathrm{p}}, B$, and $P_{\mathrm{t}}$ became more intense. An almost symmetric pressure ridge formed, and its peak coincided with the traditionally defined stream interface, although the $T_{\mathrm{p}}$ and $S$ did not increase dramatically across it. As the Sun rotated, STA encountered this SIR about one day later. The observed $V_{\mathrm{p}}$ in both the slow and fast streams increased from STB to ACE to STA, probably due to some latitudinal effects, as $\mathrm{ACE}$ at the intermediate latitude observed an intermediate peak stream speed. On the other hand, the faster stream at STA than at the other two spacecraft may be the cause for a higher 


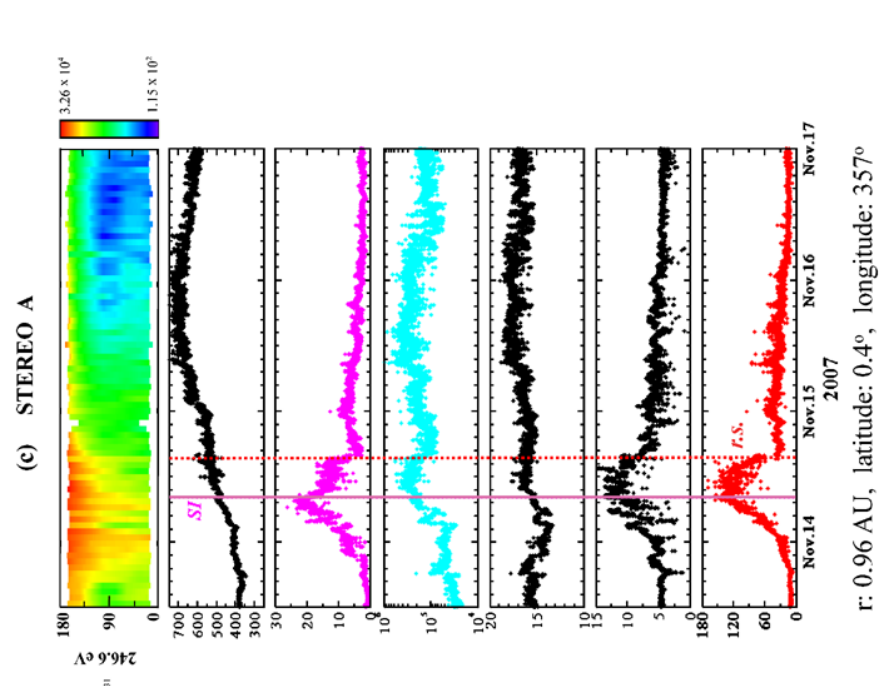

$\begin{array}{lll}\text { స. } & 0 \\ > & 0 \\ 0 & 0 & 0 \\ 0 & 0 \\ 0 & 0 \\ 0 & 0 \\ 0 & 0 \\ 0 & 0 & 0\end{array}$

过焉

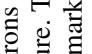

递要品

要要

结券

焉词

产这

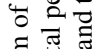

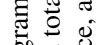

일

के 离.

월

금 흠

$\overbrace{}^{\frac{8}{3}}$

㻤

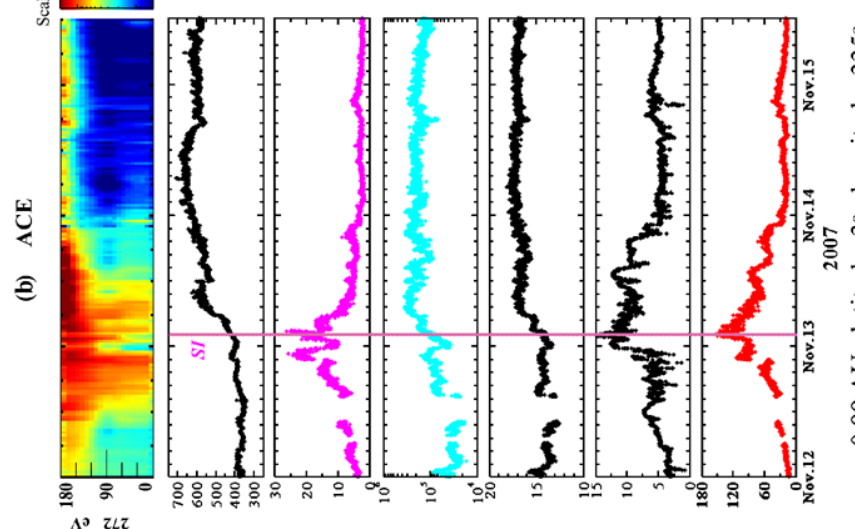

in 흄ํㅀ

으음

홍휴 뭉

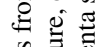

点昰

萼

Ð छ

งิ ํㅡㅇ

근

可

ว

$\Xi$ है

$\varangle$

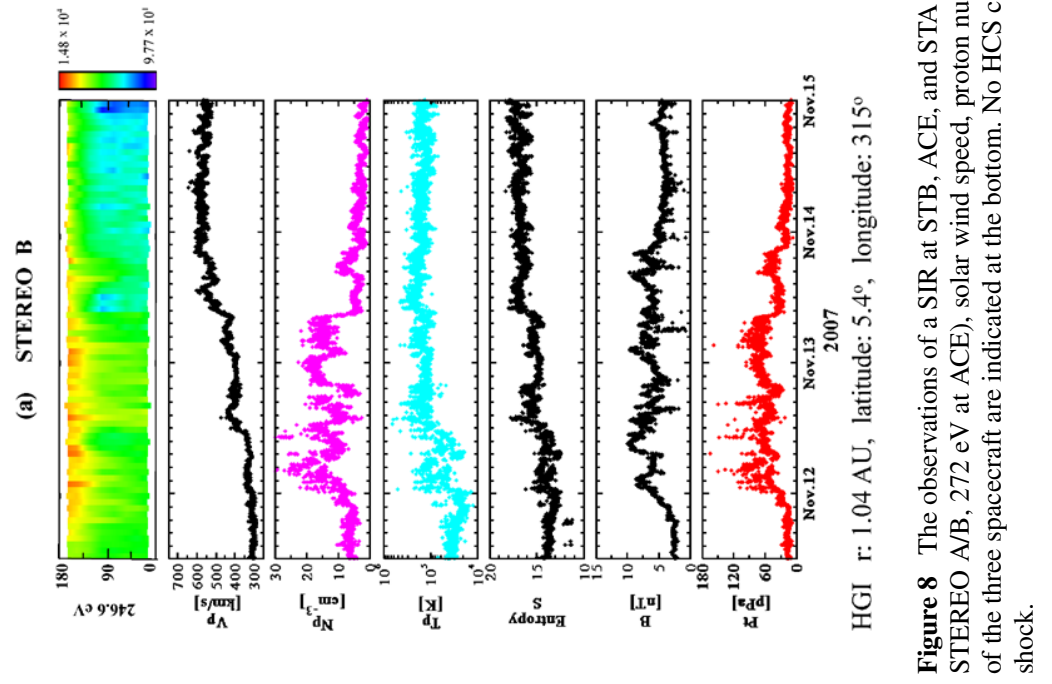


$P_{\mathrm{t}}$ peak there. The simultaneous sharp variations of the related parameters were not seen at STA, possibly due to some temporal variations of the plasma source regions and/or some dissipation within the SIR. We note that this trend is opposite to the August event, where a sharp interface was observed at STA. Therefore, we cannot simply determine the variation trends of $N_{\mathrm{p}}, T_{\mathrm{p}}$, and other parameters near the stream interface for SIRs near $1 \mathrm{AU}$. They seem to vary from case to case, probably related to the background coronal and heliospheric structures.

\section{Discussion}

In 2007, near solar minimum, the twin STEREO spacecraft along with the Wind, ACE, and Ulysses spacecraft, provided us with a much better view of SIRs than previous single point observations. In this article, we analyzed three representative SIRs in depth, as the spacecraft were separated more and more from May to August to November. The May and November events demonstrate that the solar wind parameters across the SIRs can have surprisingly similar amounts of variations, whether the spacecraft were separated by $7^{\circ}$ or $42^{\circ}$ longitude. The inherent variability in the observed SIR characteristics was noticeable even when the spacecraft were not far separated. Because multiple spacecraft rarely have exact latitudinal or longitudinal alignment, the differences in observations usually represent a combined effect of the temporal and spatial variations.

Overall, we can see that the shocks driven by SIRs at $1 \mathrm{AU}$ are still in development. They are somewhat small and transient local structures, and their directions are variable with locations. For example, in the May event, the reverse shock was missing at STB, which was separated from STA by only about $0.09 \mathrm{AU}$ in radial direction and $7^{\circ}$ longitude. From the August and November events, we see the counterstreaming suprathermal electrons upstream SIR-driven shocks are developing too.

There is no clear trend of the steepening or widening of the variations of density, temperature, and other parameters near the stream interface for SIRs around 1 AU. For example, the traditionally defined stream interface in the August event appeared at STA, the westward spacecraft, and then disappeared at Ulysses two days later. In contrast, in the November event, such simultaneous and abrupt variations of density, temperature, and other pertinent parameters were seen at STB, the eastward spacecraft, not at STA.

Related to the steepening or widening trend of the stream interface, we can see that there is still a competition between the compression and expansion effects acting over an entire SIR. In the August event, the compression effect seemed to be stronger than the expansion effect at STA; but from STA to Ulysses, the dissipation might have smoothed some abrupt variations within the SIR, and meanwhile a shock pair formed and was trying to expand the SIR at Ulysses. Also in the November event, the pressure ridge associated with the compression formed at ACE and STA, and it even steepened and formed a reverse shock at the trailing edge at STA, which was closest to the Sun.

In short, although SIRs at $1 \mathrm{AU}$ are not as dynamic as interplanetary coronal mass ejections, they indeed vary greatly with time and space. The forces acting within SIRs are not uniform and are still growing. Since all the shown SIRs herein are also long-lived CIRs, their variations also represent the variability of CIRs near 1 AU. Because CIRs usually drive periodic enhanced geomagnetic activity, our study suggests that one cannot ignore the CIR variations within short temporal and small spatial scales in order to well predict the space weather. Furthermore, we hope to compare the multi-spacecraft observations reported here with heliospheric modeling results closely in the future. We believe such a comparison can 
help to improve solar wind modeling and also synthesize our understanding of stream interactions.

Acknowledgements This research is supported by the NASA STEREO program through Grant NAS503131 administered by UC Berkeley. We sincerely thank all the PIs for making the data available. We gratefully acknowledge MWO and CCMC staff for providing us the photospheric magnetic dada and coronal modeling. We also thank R.J. Forsyth for helping us confirm Ulysses shock identifications.

Open Access This article is distributed under the terms of the Creative Commons Attribution Noncommercial License which permits any noncommercial use, distribution, and reproduction in any medium, provided the original author(s) and source are credited.

\section{References}

Acuña, M.H., Curtis, D., Scheifele, J.L., Russell, C.T., Schroeder, P., Szabó, A., Luhmann, J.G.: 2008, Space Sci. Rev. 136, 203.

Arge, C.N., Luhmann, J.G., Odstrcil, D., Schrijver, C.J., Li, Y.: 2004, J. Atmos. Solar Terr. Phys. 66, 1295.

Balogh, A., Beek, T.J., Forsyth, R.J., Hedgecock, P.C., Marquedant, R.J., Smith, E.J., Southwood, D.J., Tsurutani, B.T.: 1992, Astron. Astrophys. Suppl. 92, 221.

Balogh, A., Bothmer, V., Crooker, N.U., Forsyth, R.J., Gloeckler, G., Hewish, A., Hilchenbach, M., Kallenbach, R., Klecker, B., Linker, J.A., et al.: 1999, Space Sci. Rev. 89, 141.

Bame, S.J., McComas, D.J., Barraclough, B.L., Phillips, J.L., Sofaly, K.J., Chavez, J.C., Goldstein, B.E., Sakurai, R.K.: 1992, Astron. Astrophys. Suppl. 92, 237.

Belcher, J.W., Davis, L. Jr.: 1971, J. Geophys. Res. 76, 3534.

Crooker, N.U., Burton, M.E., Siscoe, G.L., Kahler, S.W., Gosling, J.T., Smith, E.J.: 1996, J. Geophys. Res. 101, 24331.

Feldman, W.C., Asbridge, J.R., Bame, S.J., Fenimore, E.E., Gosling, J.T.: 1981, J. Geophys. Res. 86, 5408.

Galvin, A.B., Kistler, L.M., Popecki, M.A., Farrugia, C.J., Simunac, K.D.C., Ellis, L., Möbius, E., Lee, M.A., Boehm, M., Carroll, J., et al.: 2008, Space Sci. Rev. 136, 437.

Gosling, J.T., Pizzo, V.J.: 1999, Space Sci. Rev. 89, 21.

Gosling, J.T., Asbridge, J.R., Bame, S.J., Feldman, W.C.: 1978, J. Geophys. Res. 83, 1401.

Gosling, J.T., Borrini, G., Asbridge, J.R., Bame, S.J., Feldman, W.C., Hansen, R.F.: 1981, J. Geophys. Res. 86, 5438 .

Gosling, J.T., Bame, S.J., Feldman, W.C., McComas, D.J., Phillips, J.L., Goldstein, B.E.: 1993a, Geophys. Res. Lett. 20, 2335.

Gosling, J.T., Bame, S.J., McComas, D.J., Phillips, J.L., Pizzo, V.J., Goldstein, B.E., Neugebauer, M.: 1993b, Geophys. Res. Lett. 20, 2789.

Howard, R.A., Moses, J.D., Vourlidas, A., Newmark, J.S., Socker, D.G., Plunkett, S.P., Korendyke, C.M., Cook, J.W., Hurley, A., Davila, J.M., et al.: 2008, Space Sci. Rev. 136, 67.

Hundhausen, A.J., Gosling, J.T.: 1976, J. Geophys. Res. 81, 1436.

Jian, L., Russell, C.T., Luhmann, J.G., Skoug, R.M.: 2006, Solar Phys. 239, 337.

Jian, L.K., Russell, C.T., Luhmann, J.G., Skoug, R.M., Steinberg, J.T.: 2008, Solar Phys. $249,85$.

Kaiser, M.L., Kucera, T.A., Davila, J.M., St. Cyr, O.C., Guhathakurta, M., Christian, E.: 2008, Space Sci. Rev. 136, 5 .

Lepping, R.P., Acuña, M.H., Burlaga, L.F., Farrell, W.M., Slavin, J.A., Schatten, K.H., Mariani, F., Ness, N.F., Neubauer, F.M., Whang, Y.C., et al.: 1995, Space Sci. Rev. 71, 207.

Lin, R.P., Anderson, K.A., Ashford, S., Carlson, C., Curtis, D., Ergun, R., Larson, D., McFadden, J., McCarthy, M., Parks, G.K., et al.: 1995, Space Sci. Rev. 71, 125.

Luhmann, J.G., Curtis, D.W., Schroeder, P., McCauley, J., Lin, R.P., Larson, D.E., Bale, S.D., Sauvaud, J.-A., Aoustin, C., Mewaldt, R.A., et al.: 2008, Space Sci. Rev. 136, 117.

McComas, D.J., Bame, S.J., Barker, P., Feldman, W.C., Phillips, J.L., Riley, P., Griffee, J.W.: 1998, Space Sci. Rev. 86, 563.

Ogilvie, K.W., Chornay, D.J., Fritzenreiter, R.J., Hunsaker, F., Keller, J., Lobell, J., Miller, G., Scudder, J.D., Sittler, C. Jr., Torbert, R.B., et al.: 1995, Space Sci. Rev. 71, 55.

Russell, C.T., Shinde, A.A., Jian, L.: 2005, Adv. Space Res. 35, 2178.

Russell, C.T., Jian, L.K., Blanco Cano, X., Luhmann, J.G., Zhang, T.L.: 2009, Geophys. Res. Lett. 36, L02103. doi:10.1029/2008GL036337.

Sauvaud, J.-A., Larson, D., Aoustin, C., Curtis, D., Médale, J.-L., Fedorov, A., Rouzaud, J., Luhmann, J., Moreau, T., Schröder, P., et al.: 2008, Space Sci. Rev. 136, 227. 
Smith, C.W., L’Heureux, J., Ness, N.F., Acuña, M.H., Burlaga, L.F., Scheifele, J.: 1998, Space Sci. Rev. 86, 613.

Smith, E.J., Wolfe, J.H.: 1976, J. Geophys. Res. 3, 137.

Steinberg, J.T., Gosling, J.T., Skoug, R.M., Wiens, R.C.: 2005, J. Geophys. Res. 110, A06103. doi:10.1029/ 2005JA011027.

Ulrich, R.K., Evans, S., Boyden, J.E., Webster, L.: 2002, Astrophys. J. Suppl. 139, 259.

Winterhalter, D., Smith, E.J., Burton, M.E., Murphy, N., McComas, D.J.: 1994, J. Geophys. Res. 99, A4 6667. 